\title{
Power Doppler Ultrasound Evaluation of Peripheral Joint, Entheses, Tendon, and Bursa Abnormalities in Psoriatic Patients: A Clinical Study
}

\author{
Yuanjiao Tang, Yujia Yang, Xi Xiang, Liyun Wang, Lingyan Zhang, and Li Qiu
}

\begin{abstract}
Objective. To evaluate the prevalence rates of peripheral joint, enthesis, tendon, and bursa abnormalities by power Doppler (PD) ultrasonic examination in patients with psoriatic arthritis (PsA), psoriatic patients without clinical signs of arthritis (non-PsA psoriasis group), and healthy individuals, to detect subclinical PsA.

Methods. A total of 253 healthy volunteers, 242 non-PsA psoriatic patients, and 86 patients with PsA were assessed by 2-dimensional and power Doppler (PD) ultrasound. Peripheral joint, enthesis, tendon, and bursa abnormalities were observed, characterizing abnormal PD. The affected patients and sites with abnormalities in various ages were compared among groups; PD signal grades for the abnormalities were also compared.

Results. In the PsA group, significantly higher percentages of sites showing joint effusion/synovitis, enthesitis, and tenosynovitis in all age groups, and markedly higher rates of sites with bursitis were found in young and middle age groups, compared with the non-PsA and control groups (all $\mathrm{p}<0.01$ ). Meanwhile, the non-PsA group showed significantly higher rates of joint effusion/synovitis and enthesitis sites, and elevated PD signal grades of synovitis, enthesitis, and tenosynovitis in comparison with the control group, both in young and middle age groups (all $\mathrm{p}<0.01)$.

Conclusion. Patients with PsA have high percentages and PD signal grades of peripheral joint, tendon, enthesis, and bursa involvement. Young and middle-aged non-PsA patients have high synovitis and enthesitis percentages, and elevated PD signal grades of synovitis, enthesitis, and tenosynovitis . (First Release April 15 2018; J Rheumatol 2018;45:811-17; doi:10.3899/jrheum.170765)
\end{abstract}

Key Indexing Terms:

POWER DOPPLER ULTRASOUND

PERIPHERAL ABNORMALITIES

PSORIATIC PATIENTS

Psoriatic arthritis (PsA) is a chronic inflammatory joint disease associated with psoriasis. In addition to skin and nail involvement, the clinical manifestations of PsA include dactylitis, spondylitis, and peripheral abnormalities such as synovitis, enthesitis, tenosynovitis, bursitis, and bone erosions ${ }^{1}$. PsA is deforming and destructive in $40-60 \%$ of

From the Department of Ultrasound, West China Hospital of Sichuan University, Chengdu, Sichuan, China.

Supported by grants from the National Natural Science Foundation of China (81671696 and 51703141), the Sichuan Science and Technology Plan (2015 SZ0125), and the Chengdu Science and Technology Plan (2014-HM01-00176-SF).

Y. Tang, MM, Department of Ultrasound, West China Hospital of Sichuan University; Y. Yang, BS, Department of Ultrasound, West China Hospital of Sichuan University; X. Xiang, BS, Department of Ultrasound, West China Hospital of Sichuan University; L. Wang, BS, Department of Ultrasound, West China Hospital of Sichuan University; L. Zhang, MM, Department of Ultrasound, West China Hospital of Sichuan

University; L. Qiu, MD, Department of Ultrasound, West China Hospital of Sichuan University.

Address correspondence to Dr. L. Qiu, Department of Ultrasound, West China Hospital of Sichuan University, No.37 Guo Xue Xiang, Chengdu 610041, Sichuan,China.E-mail: wsqiuli@126.com

Accepted for publication November 29, 2017. patients with consequent functional impairment, decreased quality of life, psychosocial disability, and increased risk of death $^{2,3}$. About $10 \%$ of patients with psoriasis develop PsA over a decade 4 . Therefore, it is essential to identify psoriatic patients prone to developing PsA, to ensure more aggressive treatments and prevent irreversible joint damage.

Previous studies have revealed a high frequency of peripheral abnormalities in psoriatic patients without musculoskeletal symptoms $5,6,7,8,9$. Subclinical changes in joints, entheses, and tendons may occur before the onset of PsA, and likely occur in a large proportion of psoriatic patients ${ }^{10}$. Previous findings indicate that synovitis and enthesitis are more frequent in psoriatic patients with no musculoskeletal diseases compared with healthy subjects. Therefore, these manifestations may be early signs of PsA ${ }^{4,11,12}$. Meanwhile, subclinical inflammatory lesions are highly prevalent in patients with cutaneous psoriasis ${ }^{13}$. According to previous studies, changes in joints, entheses, and tendons are affected by age and body mass index (BMI). With increasing age or BMI, peripheral abnormalities (e.g., enthesitis) are more common in normal asymptomatic subjects or patients with psoriasis $^{4,8,14}$. Moshrif, et $a l^{8}$ found statistically significant

Personal non-commercial use only. The Journal of Rheumatology Copyright $\subset$ 2018. All rights reserved. 
positive associations of enthesitis occurrence with patient age and BMI. In addition, Milella, et $a l^{15}$ indicated that entheseal morphology primarily reflects the age of an individual. Further, Andrade and Barbosa-Branco ${ }^{16}$ showed that prevalence and duration of work disability as a result of synovitis and tenosynovitis are associated with the age factor. In enthesitis, other confounding factors such as higher mechanical stress could also affect entheseal changes. The synovioentheseal complex (SEC) is a very specialized region in some entheseal organs with fibrocartilage lining the enthesis. It provides oxygen and nutrients for the fibrocartilage and removes waste products, maintaining the normal function of the whole entheseal organ. The inflammatory microenvironment of the SEC is called enthesitis. Fibrocartilages are often located inside the joints or intraarticular areas, partly forming structures called the bursa, which is lined by the synovium. A problem arising in fibrocartilages could cause inflammation in the adjacent synovium, a phenomenon referred to as bursitis. Patients with PsA seek medical attention with joint swelling that is sometimes a result of primary abnormalities of the SEC.

Ultrasound (US) is considered a more reliable and sensitive technique to assess both disease activity and damage in inflammatory arthritis, including PsA, compared with physical examination ${ }^{17,18,19}$. This imaging technique is proven to be a powerful diagnostic tool in the detection and measurement of synovial inflammation, as well as enthesitis, tenosynovitis, and bursitis evaluation in PsA. US results are comparable to those of magnetic resonance imaging (MRI), except for bone marrow edema detection. Reproducibility and low cost of US provide advantages over MRI for PsA detection ${ }^{20,21,22,23}$. With power Doppler (PD), US enables very sensitive visualization of blood flow at the synovium, entheses, tendon, and bursa levels, and evaluates the inflammatory activity. According to Zabotti, et al $^{19}$, further research is required for the use of US in the management of psoriatic disease.

The main aim of our present study was to determine the prevalence rates of peripheral joint, entheses, tendon, and bursa abnormalities by means of PDUS examination in patients with PsA, psoriatic patients without the clinical signs of arthritis (non-PsA psoriasis group), and healthy individuals of different age groups, to identify patients with subclinical PsA requiring more attention and to prevent PsA.

\section{MATERIALS AND METHODS}

Patient population. This cross-sectional study comprised 581 psoriatic patients and healthy volunteers aged $\geq 18$ years. They included 242 psoriatic patients without clinical signs of arthritis (non-PsA group), 86 patients with PsA (PsA group) from the Ultrasound Department, and 253 healthy volunteers (control group) from the physical examination center at West China Hospital, Sichuan University, from January 2014 to June 2016. All participants were randomly selected. The study was approved by the ethics committee of West China Hospital, Sichuan University (approval number: ChiCTR-DCD-15006851). All subjects provided informed consent for scientific evaluation of their imaging data at the time of examination.
The inclusion criterion for the non-PsA group was psoriasis diagnosed pathologically based on skin biopsy by a dermatologist. Exclusion criteria were the following: (1) pregnant women; (2) a recent history of trauma; (3) engagement in heavy manual work; (4) PsA diagnosis or a history of other forms of arthritis; and (5) current or recent ( $\leq 3 \mathrm{mos}$ ) systemic treatment for psoriasis. For the PsA group, inclusion criterion was compliance with Classification Criteria for Psoriasis Arthritis diagnostic criteria. Exclusion criteria were the following: (1) pregnancy; (2) recent history of trauma; (3) engagement in heavy manual work; (4) a history of any other form of arthritis; and (5) current or recent ( $\leq 3$ months) systemic treatment for psoriasis. For the control group, exclusion criteria were as follows: (1) pregnant women; (2) a recent history of trauma; (3) engagement in heavy manual work; and (4) a current or previous history of any dermal disease or any form of arthritis.

Based on age, the subjects were divided into 3 groups: youth (18-44 yrs), middle-aged (45-59 yrs), and elderly ( $\geq 60 \mathrm{yrs}$ ) groups.

US examination. The joints, entheses, tendons, and bursae of 10 psoriatic patients were evaluated by 2 radiologists, and joint effusion/synovitis-, enthesitis-, tenosynovitis-, and bursitis-related changes in 2-dimensional and PDUS were observed. Two-dimensional PDUS (Philips IU22) was used to assess peripheral joints, entheses, tendons, and bursae in each subject. Probe frequency was set at 3-9 MHz for hip evaluation, and at 5-12 $\mathrm{MHz}$ to examine other peripheral joints and the musculoskeletal condition selected. Gain was set at maximum sensitivity without noise signals. The joints scanned included the shoulders, elbows, wrists, hips, knees, ankles, metacarpophalangeal and interphalangeal joints of the fingers, and metatarsophalangeal and interphalangeal joints of the toes (a total of 78 joints per patient). The entheses scanned included common flexor and extensor entheses of hands, quadriceps, proximal and distal patellar, Achilles, and plantar aponeurosis entheses (a total of 14 per patient). The tendons scanned included the long heads of biceps brachii, flexor and extensor tendons of hands and feet, anterior tibial muscle, tibialis posterior, and peroneal tendon sheaths (a total of 42 per individual). The bursae scanned included the retrocalcaneal, subacromial-subdeltoid, and trochanteric bursae (a total of 6 per individual). All joints and bursae underwent scanning from the front, rear, inner, and outer sides. All entheses and tendons underwent transverse and longitudinal complete scanning. Joint effusion/synovitis, enthesitis, tenosynovitis, and bursitis were observed. PDUS was used to detect blood supply in these abnormalities.

The patients were diagnosed by the following US findings: (1) joint effusion was reflected by an echoless zone in the joint cavity, with no blood flow signal; (2) synovitis was detected by a hypoechoic signal in the joint capsule and no thinning under pressure, with or without visible points of linear flow signals within the synovium (Figure 1A); (3) enthesitis was identified according to the Outcome Measures in Rheumatology clinical trials definition ${ }^{24}$ [i.e., abnormal hypoechogenicity or loss of normal fibrillar architecture, thickened tendon at the bone attachment (may occasionally contain hyperechogenicity foci consistent with calcification)], in 2 perpendicular planes, with possible bony changes, including enthesophytes, erosions, or irregularity, and the presence of only 1 of the above features with or without Doppler signal was defined as enthesitis (Figure 1C); (4) tenosynovitis was reflected by hypoechoic or anechoic thickened tissues with or without fluid within the tendon sheath, in 2 perpendicular planes with possible Doppler signals (Figure 1E); and (5) bursitis was diagnosed by a widening echoless zone or synovial thickening area at the site of an anatomical bursa.

Semiquantitative findings of PDUS activity for synovitis, tenosynovitis, and bursitis were scored as follows: Grade 0 (no intraarticular color signal), grade 1 ( $\leq 3$ color signals representing only low flow), grade $2(<50 \%$ of the intraarticular area filled with color signals representing clear flow), grade 3 ( $>50 \%$ of the intraarticular area filled with color signals ${ }^{25}$; Figure 1B and Figure 1F). PDUS activity for enthesitis was graded as follows: no flow signal (grade 0) and flow signal (grade 1; Figure 1D).

US scan was performed by 2 radiologists with 7 and 10 years' experience in musculoskeletal US imaging. The scanning time was about 30 to $60 \mathrm{~min}$

Personal non-commercial use only. The Journal of Rheumatology Copyright $@$ 2018. All rights reserved. 

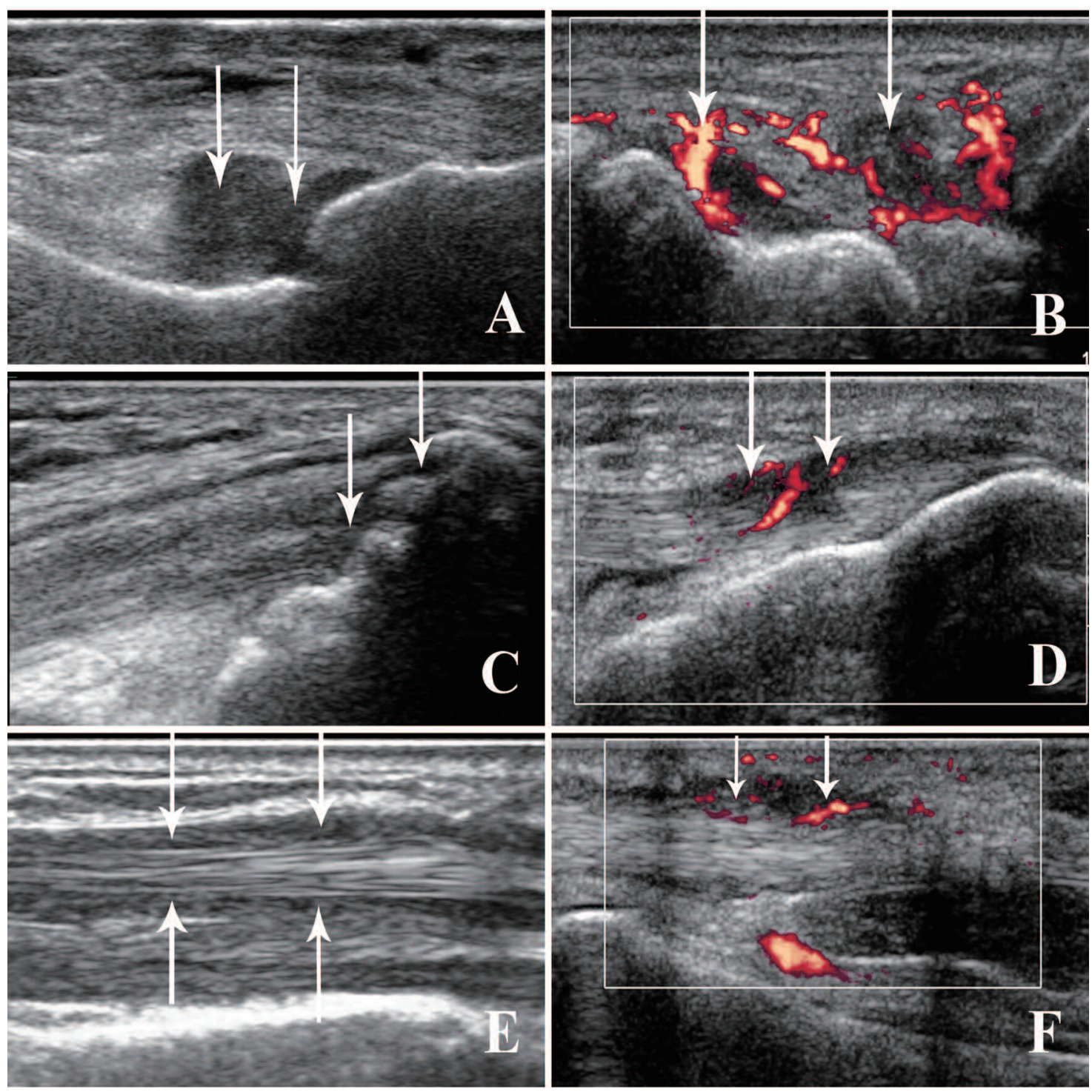

Figure 1. Two-dimensional and PD ultrasonic appearances of synovitis, enthesitis, tenosynovitis, and bursitis. A. Synovitis in a left metatarsophalangeal joint in a non-PsA psoriatic patient. Arrows indicate the thickened synovium. B. Synovitis in a right wrist in a PsA patient with PD grade 3. Arrows indicate the thickened synovium and its rich blood flow. C. Enthesitis in a left femoral quadriceps tendon in a non-PsA psoriatic patient. Arrows indicate the hypoechogenicity of the thickened tendon at the bone attachments, which contain hyperechogenicity foci. D. Enthesitis in a right patella ligament in a PsA patient with PD grade 1. Arrows indicate the hypoechogenicity of the thickened ligament at the distal bone attachments, which exhibit rich Doppler signal. E. Tenosynovitis in an extensor tendon of the left hand in a patient with PsA. Arrows indicate the hypoechoic thickened tissue within the tendon sheath. F. Tenosynovitis in a flexor tendon of the right hand in a PsA patient with PD grade 2. Arrows indicate the hypoecho at peritendon, which exhibits a rich Doppler signal. PD: power Doppler; PsA: psoriatic arthritis.

per individual, depending on peripheral abnormalities. The imaging experts were blinded to patient data; all patients and healthy subjects underwent US in a random order and were instructed not to communicate with the sonographer about their disease during the US assessment. Before the study, the investigators reached a consensus on the US scanning technique to adopt and data interpretation. A diagnosis consistency test was conducted, and a $\mathrm{\kappa}$ value of 0.85 was obtained.

Statistical analysis. The SPSS 19.0 (SPSS Inc.) software was used for data analysis, with $\mathrm{p}<0.05$ considered statistically significant. Measurement data were compared by 1-way ANOVA or sample t test. Enumeration data were compared by Pearson chi-square test or Fisher's exact test, with a Bonferroni correction. Ranked data were assessed by the Kruskal-Wallis $\mathrm{H}$ test or Mann-Whitney U test, also with a Bonferroni correction.

\section{RESULTS}

Patient characteristics. Our current study included 581 patients and healthy volunteers aged $\geq 18$ years. Based on age, the participants were divided into 3 groups: non-PsA, PsA, and control groups. The main characteristics of the

\section{Personal non-commercial use only. The Journal of Rheumatology Copyright @ 2018. All rights reserved.}


study patients are shown in Table 1. Age, sex distribution, and body mass index (BMI) among groups showed no significant differences. The duration of psoriasis was significantly longer in patients with PsA compared with the non-PsA group $(\mathrm{p}<0.05)$.

Comparison of affected patients with joint effusion/synovitis among various age groups. A significantly higher percentage of patients with PsA showed joint effusion/synovitis compared with non-PsA patients and healthy controls, in young and middle-aged groups (all $\mathrm{p}<0.01$ ). In addition, a higher percentage of non-PsA patients showed joint effusion/synovitis compared with healthy controls in the middle-aged group $(\mathrm{p}<0.01)$. However, there were no significant differences in joint effusion/synovitis in elderly patients of different groups (Table 2).

Comparisons of affected sites among patients with joint effusion/synovitis in various age groups. In the PsA group, a significantly higher percentage of sites with joint effusion/synovitis was observed, compared with the non-PsA and control groups for all age groups (all $\mathrm{p}<0.01$ ). In addition, significantly higher percentages of sites in the non-PsA group showed abnormalities compared with control patients, both in the young and middle-aged groups (both $\mathrm{p}<0.01$; Table 2).

Comparisons of PD signal grades of synovitis among patients with joint effusion/synovitis in various age groups. Significantly higher PD signal grades of synovitis in the PsA group were found, compared with the control and non-PsA groups, for all age groups (all $\mathrm{p}<0.01$ ). Meanwhile, PD signal grades of synovitis in the non-PsA group were significantly higher than those of control patients, in the young and middle-aged groups (both $\mathrm{p}<0.01$; Table 2).

Comparison of affected patients with enthesitis among various age groups. A significantly higher percentage of patients with PsA had enthesitis compared with non-PsA patients and healthy controls for the young age groups $(\mathrm{p}<0.01)$. Meanwhile, there were no significant differences in enthesitis among the groups, both for the middle-aged and elderly groups. In the elderly individuals, enthesitis had high incidence rates in all 3 study groups (Table 3 ).

Comparisons of affected sites among patients with enthesitis in various age groups. In the PsA group, there were significantly higher percentages of sites showing enthesitis compared with the non-PsA and control groups in all age groups (all $\mathrm{p}<0.01$ ). In addition, the non-PsA group showed a significantly higher percentage of sites with enthesitis compared with control patients, both for the young and middle-aged groups (both $\mathrm{p}<0.01$; Table 3 ).

Comparisons of PD signal grades of enthesitis among various age groups. Compared with the non-PsA and control groups, the patients with PsA showed significantly higher percentages of PD signal grades of enthesitis for all ages (all $\mathrm{p}<0.01)$. Meanwhile, the PD signal grades of enthesitis in the non-PsA group were significantly higher than those of

Table 1. Patient characteristics based on age. Values are mean \pm SD unless otherwise specified.

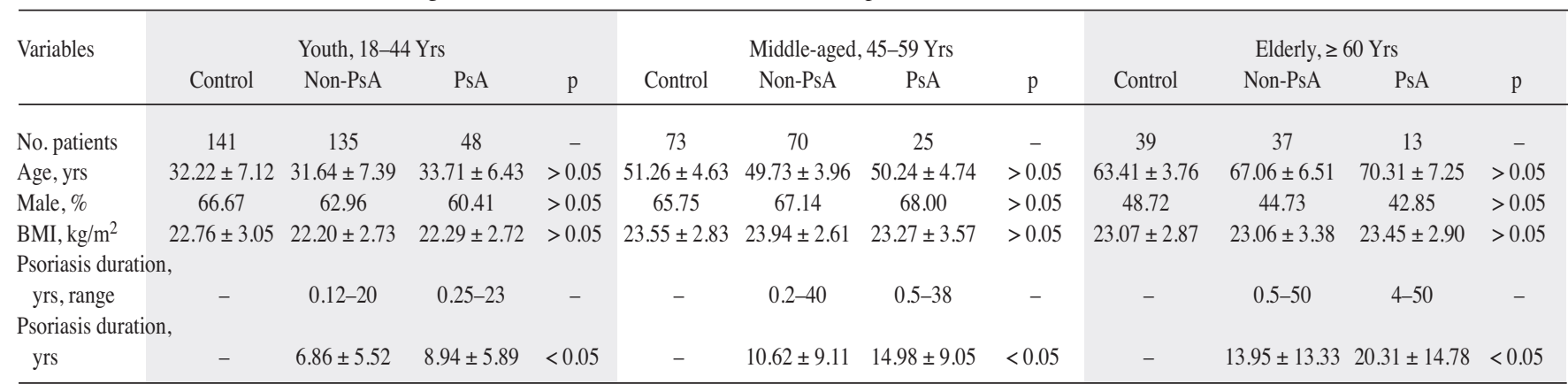

PsA: psoriatic arthritis; BMI: body mass index.

Table 2. Comparisons of joint effusion/synovitis among groups, based on age. Values are $\mathrm{n}(\%)$ unless otherwise specified.

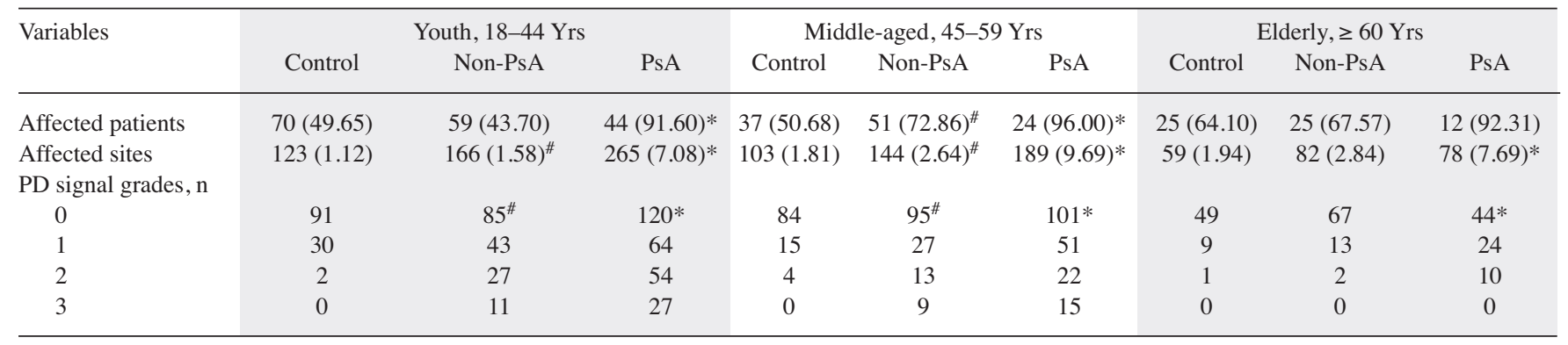

$* \mathrm{p}<0.01$ versus control and non-PsA groups. ${ }^{\#} \mathrm{p}<0.01$ versus control group. PsA: psoriatic arthritis; PD: power Doppler. 
Table 3. Comparison of enthesitis among groups, based on age. Values are $\mathrm{n}(\%)$ unless otherwise specified.

\begin{tabular}{|c|c|c|c|c|c|c|c|c|c|}
\hline \multirow[t]{2}{*}{ Variables } & \multicolumn{3}{|c|}{ Youth, 18-44 Yrs } & \multicolumn{3}{|c|}{ Middle-aged, 45-59 Yrs } & \multicolumn{3}{|c|}{ Elderly, $\geq 60$ Yrs } \\
\hline & Control & Non-PsA & PsA & Control & Non-PsA & PsA & Control & Non-PsA & PsA \\
\hline Affected patients & $48(34.00)$ & $55(40.74)$ & $37(77.08)^{*}$ & $42(57.53)$ & $47(67.14)$ & $19(76.00)$ & $30(76.92)$ & $31(83.78)$ & $11(84.62)$ \\
\hline \multicolumn{10}{|c|}{ PD signal grades, $\mathrm{n}$} \\
\hline 0 & 104 & $129^{\#}$ & $63^{*}$ & 119 & $140^{\#}$ & $76^{*}$ & 91 & 99 & $58 *$ \\
\hline 1 & 0 & 27 & 29 & 0 & 30 & 35 & 0 & 4 & 7 \\
\hline
\end{tabular}

${ }^{*} \mathrm{p}<0.01$ versus control and non-PsA groups. ${ }^{\#} \mathrm{p}<0.01$ versus control group. PsA: psoriatic arthritis; PD: power Doppler.

control patients, in the young and middle-aged groups (both $\mathrm{p}<0.01)$. Of note, none of the controls showed PD signal grades of enthesitis (Table 3).

Comparison of affected patients with tenosynovitis among various age groups. A significantly higher percentage of patients with PsA showed tenosynovitis compared with non-PsA patients and healthy individuals, for all age groups (all $\mathrm{p}<0.01$ ). However, there were no significant differences of affected patients showing tenosynovitis among non-PsA patients and healthy controls in all age groups (Table 4).

Comparisons of affected sites among patients with tenosynovitis in various age groups. In the PsA group, a significantly higher percentage of sites showed enthesitis compared with the non-PsA and control groups, in all age groups (all $\mathrm{p}<0.01)$. However, there were no significant differences of sites with tenosynovitis between the non-PsA and control groups, in all age groups (Table 4).

Comparisons of PD signal grades of tenosynovitis among various age groups. PD signal grades of tenosynovitis in the PsA group were significantly higher than those of the non-PsA and control groups for the young age group and elevated compared with control patients for the middle-aged group (all $\mathrm{p}<0.01$ ). Meanwhile, the non-PsA group showed a significantly higher percentage of PD signal grades of tenosynovitis compared with control patients, for young and middle-aged individuals (both $\mathrm{p}<0.01$ ). However, PD signal grades of tenosynovitis among various groups showed no significant differences for the elderly age group (Table 4).

Comparison of affected patients with bursitis among various age groups. A significantly higher percentage of patients with PsA showed bursitis compared with non-PsA patients and healthy individuals, for the young and middle-aged groups (both $\mathrm{p}<0.01$ ). However, there were no significant differences in affected patients showing bursitis among study groups in the elderly population, and between the non-PsA and control groups in all age groups (Table 5).

Comparisons of affected sites among patients with bursitis in various age groups. In the PsA group, a significantly higher percentage of sites showed bursitis compared with the non-Ps A and control groups, for the young and middle-aged groups (both $\mathrm{p}<0.01$ ). However, no significant differences of sites with bursitis were found among study groups in elderly individuals, and between the non-PsA and control groups, in all age groups (Table 5).

Comparisons of PD signal grades of bursitis among various age groups. Compared with the non-PsA and control groups, patients with PsA showed significantly higher PD signal grades of bursitis, for the young age group (both $\mathrm{p}<0.01$ ). However, no significant differences were found among study groups in the middle-aged and older groups, and between the non-PsA and control groups, in all age groups (Table 5).

\section{DISCUSSION}

In our study, the duration of psoriasis in patients with PsA was significantly longer than that of the non-PsA group. We speculate that non-PsA psoriatic patients may develop PsA with time.

In psoriatic patients, especially those with PsA, inflam-

Table 4. Comparison of tenosynovitis among groups, based on age. Values are $\mathrm{n}(\%)$ unless otherwise specified.

\begin{tabular}{|c|c|c|c|c|c|c|c|c|c|}
\hline Variables & Control & Non-PsA & PsA & Control & Non-PsA & PsA & Control & Non-PsA & PsA \\
\hline Affected patients & $4(2.83)$ & $7(5.19)$ & $19(39.58)^{*}$ & $3(4.10)$ & $9(12.86)$ & $15(60.00)^{*}$ & $6(15.38)$ & $7(18.92)$ & $4(2.83)$ \\
\hline \multicolumn{10}{|l|}{ PD signal grades, $\mathrm{n}$} \\
\hline 0 & 8 & $3^{\#}$ & $2 *$ & 32 & $2^{\#}$ & $15^{\#}$ & 9 & 10 & 10 \\
\hline 1 & 0 & 5 & 9 & 2 & 6 & 26 & 0 & 1 & 3 \\
\hline
\end{tabular}

$* \mathrm{p}<0.01$ versus control and non-PsA groups. ${ }^{\#} \mathrm{p}<0.01$ versus control group. PsA: psoriatic arthritis; PD: power Doppler.

$$
\text { Personal non-commercial use only. The Journal of Rheumatology Copyright @ } \text { 2018. All rights reserved. }
$$


Table 5. Comparison of bursitis among groups, based on age. Values are $\mathrm{n}(\%)$ unless otherwise specified.

\begin{tabular}{|c|c|c|c|c|c|c|c|c|c|}
\hline \multirow[t]{2}{*}{ Variables } & \multicolumn{3}{|c|}{ Youth, 18-44 Yrs } & \multicolumn{3}{|c|}{ Middle-aged, 45-59 Yrs } & \multicolumn{3}{|c|}{ Elderly, $\geq 60$ Yrs } \\
\hline & Control & Non-PsA & PsA & Control & Non-PsA & PsA & Control & Non-PsA & PsA \\
\hline Affected patients & $4(2.84)$ & $5(3.70)$ & $15(31.25)^{*}$ & $2(2.74)$ & $3(4.29)$ & $8(32.00)^{*}$ & $3(7.69)$ & $3(8.11)$ & $4(2.84)$ \\
\hline \multicolumn{10}{|l|}{ PD signal grades, $\mathrm{n}$} \\
\hline 0 & 5 & 2 & $5^{\#}$ & 3 & 4 & 3 & 3 & 3 & 2 \\
\hline 1 & 0 & 5 & 13 & 0 & 0 & 4 & 0 & 0 & 1 \\
\hline
\end{tabular}

${ }^{*} \mathrm{p}<0.01$ versus control and non-PsA groups. ${ }^{\#} \mathrm{p}<0.01$ versus control group. PsA: psoriatic arthritis; PD: power Doppler.

matory lesions can be axial (sacroiliitis and spondylitis) or peripheral (peripheral joint synovitis, enthesitis, tenosynovitis, and bursitis). Such lesions can develop without symptoms and signs, and be overlooked by both patient and physician ${ }^{26,27}$. Our study assessed various peripheral inflammatory lesions based on patient age.

We found the following in all ages: (1) patients with PsA had higher percentages and PD signal grades of synovitis compared with non-PsA psoriatic patients and healthy controls; (2) enthesitis occurred in higher percentages of patients and sites, and higher PD signal grades of enthesitis were obtained in patients with PsA compared with non-PsA and control groups; (3) higher percentages of tenosynovitis were found in patients and sites in PsA compared with the non-PsA and control groups. These findings were consistent with the clinical manifestations of patients with PsA who show more pain, dyskinesia, dysfunction, as well as worse quality of life, compared with non-PsA psoriatic patients and healthy individuals. Our results also corroborate a report demonstrating that patients with PsA have higher PD of enthesitis compared with ordinary patients with psoriasis, suggesting increased inflammatory process in $\mathrm{PsA}^{28}$. Bandinelli, et $a l^{29}$ found that US detects subclinical entheseal involvement in early PsA. Based on the SEC theory, when a mechanically stressed enthesis is injured, any associated inflammatory reaction would be expected to manifest prominently within the juxtaposed synovium. The central tenet of the SEC theory is that primary entheseal abnormalities trigger secondary joint synovitis. The SEC is a unifying factor in understanding PsA-associated synovitis. The blood vessels of entheses may originate from the underlying bone at sites with no disease onset on the subchondral bone plate; alternatively, they may invade from tendon surface tissues, including the synovium ${ }^{30}$. In all ages, none of the healthy controls showed PD signal grades of enthesitis; this is in line with previous findings ${ }^{11}$. This indicates that enthesitis shows low inflammatory activity in healthy individuals, regardless of age.

Importantly, there were several findings regarding the young and middle-aged groups. First, non-PsA psoriatic patients had higher percentage and PD signal grades of synovitis compared with healthy controls. Therefore, in these age groups, non-PsA psoriatic patients tend to have subclinical synovitis, which may even have higher inflammatory activity. In agreement, a previous study demonstrated that the prevalence of asymptomatic PDUS synovitis is significantly higher in psoriatic patients than in healthy controls ${ }^{11}$. Second, enthesitis occurred in a higher percentage of sites with elevated PD signal grades in the non-PsA group compared with control patients. These findings indicated that both greyscale US and PD data indicative of enthesitis are more common in non-PsA psoriatic patients than healthy individuals, and that these data could predict the clinical onset of PSA in these age groups, as proposed previously ${ }^{6,11,31}$. Third, PD signal grades of tenosynovitis in the non-PsA group were higher than control values. This indicated that non-PsA psoriatic patients in these age groups have active inflammatory reactions of tenosynovitis, which might be overlooked by the clinician. Fourth, bursitis was found in higher percentages of patients and sites in the PsA group compared with the non-PsA and control groups. These findings indicate that bursitis should be taken into account in PsA patients, especially in these age groups. On one hand, enthesitis can cause bursitis because of the SEC. On the other hand, bursitis is also an inflammatory process in PsA. Based on the SEC theory, in healthy individuals, the synovium plays a pivotal role in the nourishment and lubrication of the entheseal fibrocartilage, in a manner identical to its function for the articular cartilage. In disease conditions, however, this close functional interdependence could lead to bursitis ${ }^{30}$.

In the elderly group, there were no significant differences between the non-PsA and control groups in patient and site percentages of enthesitis as well as PD signal grades. This may be because pathologic processes leading to enthesitis, such as metabolic, inflammatory, traumatic, and degenerative processes, increase in incidence with time.

Our study has some limitations. First, our study did not compare US findings with MRI data. Second, other abnormalities of psoriasis, such as peritendinous edema, other osseous phenotypes, dactylitis, and nail changes were not assessed despite good sensitivity of US. These limitations should be addressed in future studies.

Personal non-commercial use only. The Journal of Rheumatology Copyright @ 2018. All rights reserved 
Overall, patients with PsA show high percentages and PD signal grades of peripheral joint, tendon, entheses, and bursa involvement. Further, our results suggest that non-PsA psoriatic patients of young and middle-aged groups have high percentages of synovitis and enthesitis, as well as elevated PD signal grades of synovitis, enthesitis, and tenosynovitis, which may indicate subclinical musculoskeletal involvement, predicting the clinical onset of PsA in these age groups. Therefore, regular US examination is quite important for patients with PsA, and more attention should be paid to young and middle-aged non-PsA psoriatic patients.

\section{REFERENCES}

1. Cozzi F, Raffeiner B, Beltrame V, Ciprian L, Coran A, Botsios C. Effects of mud-bath therapy in psoriatic arthritis patients treated with TNF inhibitors. Clinical evaluation and assessment of synovial inflammation by contrast-enhanced ultrasound (CEUS). Joint Bone Spine 2015;82:104-8.

2. McHugh NJ, Balachrishnan C, Jones SM. Progression of peripheral joint disease in psoriatic arthritis: a 5-yr prospective study. Rheumatology 2003;42:778-83.

3. D'Angelo S, Palazzi C, Gilio M, Leccese P, Padula A, Olivieri I. Improvements in diagnostic tools for early detection of psoriatic arthritis. Expert Rev Clin Immunol 2016;12:1209-15.

4. Delle Sedie A, Riente L. Psoriatic arthritis: what ultrasound can provide us. Clin Exp Rheumatol 2015;33:S60-5.

5. Scarpa R, Cuocolo A, Peluso R, Atteno M, Gisonni P, Iervolino S, et al. Early psoriatic arthritis: the clinical spectrum. J Rheumatol 2008;35:137-41.

6. Tinazzi I, McGonagle D, Biasi D, Confente S, Caimmi C, Girolomoni G, et al. Preliminary evidence that subclinical enthesopathy may predict psoriatic arthritis in patients with psoriasis. J Rheumatol 2011;38:2691-2.

7. Acquacalda E, Albert C, Montaudie H, Fontas E, Danre A, Roux $\mathrm{CH}$, et al. Ultrasound study of entheses in psoriasis patients with or without musculoskeletal symptoms: A prospective study. Joint Bone Spine 2015;82:267-71.

8. Moshrif A, Mosallam A, Mohamed EE, Gouda W, Doma M. Subclinical enthesopathy in patients with psoriasis and its association with other disease parameters: a power Doppler ultrasonographic study. Eur J Rheumatol 2017;4:24-8.

9. Gisondi P, Tinazzi I, El-Dalati G, Gallo M, Biasi D, Barbara LM, et al. Lower limb enthesopathy in patients with psoriasis without clinical signs of arthropathy: a hospital-based case-control study. Ann Rheum Dis 2008;67:26-30.

10. Simon D, Faustini F, Kleyer A, Haschka J, Englbrecht M, Kraus S, et al. Analysis of periarticular bone changes in patients with cutaneous psoriasis without associated psoriatic arthritis. Ann Rheum Dis 2016;75:660-6.

11. Naredo E, Möller I, de Miguel E, Batlle-Gualda E, Acebes C, Brito E, et al; Ultrasound School of the Spanish Society of Rheumatology and Spanish ECO-APs Group. High prevalence of ultrasonographic synovitis and enthesopathy in patients with psoriasis without psoriatic arthritis: a prospective case-control study. Rheumatology 2011;50:1838-48.

12. Emad Y, Ragab Y, Gheita T, Anbar A, Kamal H, Saad A, et al; Knee Enthesitis Working Group. Knee enthesitis and synovitis on magnetic resonance imaging in patients with psoriasis without arthritic symptoms. J Rheumatol 2012;39:1979-86.

13. Faustini F, Simon D, Oliveira I, Kleyer A, Haschka J, Englbrecht M, et al. Subclinical joint inflammation in patients with psoriasis without concomitant psoriatic arthritis: a cross-sectional and longitudinal analysis. Ann Rheum Dis 2016;75:2068-74.
14. Listi GA. The use of entheseal changes in the femur and os coxa for age assessment. J Forensic Sci 2016;61:12-8.

15. Milella M, Giovanna Belcastro M, Zollikofer CP, Mariotti V. The effect of age, sex, and physical activity on entheseal morphology in a contemporary Italian skeletal collection. Am J Phys Anthropol 2012;148:379-88

16. Andrade DM, Barbosa-Branco A. Synovitis and tenosynovitis in Brazil: analysis of sickness benefit claims. Rev Bras Epidemiol 2015;18:194-207.

17. Weiner SM, Jurenz S, Uhl M, Lange-Nolde A, Warnatz K, Peter $\mathrm{HH}$, et al. Ultrasonography in the assessment of peripheral joint involvement in psoriatic arthritis: a comparison with radiography, MRI and scintigraphy. Clin Rheumatol 2008;27:983-9.

18. Wiell C, Szkudlarek M, Hasselquist M, Møller JM, Vestergaard A, Nørregaard J, et al. Ultrasonography, magnetic resonance imaging, radiography, and clinical assessment of inflammatory and destructive changes in fingers and toes of patients with psoriatic arthritis. Arthritis Res Ther 2007;9:R119.

19. Zabotti A, Bandinelli F, Batticciotto A, Scirè CA, Iagnocco A, Sakellariou G; Musculoskeletal Ultrasound Study Group of the Italian Society of Rheumatology. Musculoskeletal ultrasonography for psoriatic arthritis and psoriasis patients: a systematic literature review. Rheumatology 2017;56:1518-32.

20. Gutierrez M, Filippucci E, De Angelis R, Filosa G, Kane D, Grassi W. A sonographic spectrum of psoriatic arthritis: "the five targets". Clin Rheumatol 2010;29:133-42.

21. Sakkas LI, Alexiou I, Simopoulou T, Vlychou M. Enthesitis in psoriatic arthritis. Semin Arthritis Rheum 2013;43:325-34.

22. Taljanovic MS, Melville DM, Gimber LH, Scalcione LR, Miller MD, Kwoh CK, et al. High-resolution US of rheumatologic diseases. Radiographics 2015;35:2026-48.

23. Coates LC, Hodgson R, Conaghan PG, Freeston JE. MRI and ultrasonography for diagnosis and monitoring of psoriatic arthritis. Best Pract Res Clin Rheumatol 2012;26:805-22.

24. Wakefield RJ, Balint PV, Szkudlarek M, Filippucci E, Backhaus M, D'Agostino MA, et al; OMERACT 7 Special Interest Group. Musculoskeletal ultrasound including definitions for ultrasonographic pathology. J Rheumatol 2005;32:2485-7.

25. Backhaus M, Ohrndorf S, Kellner H, Strunk J, Backhaus TM, Hartung W, et al. Evaluation of a novel 7-joint ultrasound score in daily rheumatologic practice: a pilot project. Arthritis Rheum 2009;61:1194-201.

26. Erdem CZ, Tekin NS, Sarikaya S, Erdem LO, Gulec S. MR imaging features of foot involvement in patients with psoriasis. Eur J Radiol 2008;67:521-5

27. Palazzi C, Lubrano E, D’Angelo S, Olivieri I. Beyond early diagnosis: occult psoriatic arthritis. J Rheumatol 2010;37:1556-8.

28. Aydin SZ, Ash ZR, Tinazzi I, Castillo-Gallego C, Kwok C, Wilson $\mathrm{C}$, et al. The link between enthesitis and arthritis in psoriatic arthritis: a switch to a vascular phenotype at insertions may play a role in arthritis development. Ann Rheum Dis 2013;72:992-5.

29. Bandinelli F, Prignano F, Bonciani D, Bartoli F, Collaku L, Candelieri A, et al. Ultrasound detects occult entheseal involvement in early psoriatic arthritis independently of clinical features and psoriasis severity. Clin Exp Rheumatol 2013;31:219-24.

30. McGonagle D, Lories RJ, Tan AL, Benjamin M. The concept of a "synovio-entheseal complex" and its implications for understanding joint inflammation and damage in psoriatic arthritis and beyond. Arthritis Rheum 2007;56:2482-91.

31. Gutierrez M, Filippucci E, De Angelis R, Salaffi F, Filosa G, Ruta S, et al. Subclinical entheseal involvement in patients with psoriasis: an ultrasound study. Semin Arthritis Rheum 2011;40:407-12. 